Cite this: Nanoscale, 2014, 6, 5251

\title{
Attenuation of encrustation by self-assembled inorganic fullerene-like nanoparticles $\uparrow$
}

\author{
Racheli Ron, ${ }^{a}$ David Zbaida, ${ }^{a}$ Ilan Z. Kafka, ${ }^{\text {b }}$ Rita Rosentsveig, ${ }^{a}$ Ilan Leibovitch $^{\text {bc }}$ \\ and Reshef Tenne*a
}

Ureteral stents and urethral catheters are commonly used medical devices for maintaining urinary flow. However, long-term placement (>30 days) of these devices in the urinary tracts is limited by the development of encrustation, a phenomenon that holds a prevalence of $50 \%$ within this patient population, resulting in a great deal of morbidity to the patients. Here we report the influence of surface coating of an all-silicone catheter with rhenium-doped fullerene-like molybdenum disulfide (Re:IF-MoS 2 ) nanoparticles on the growth and attachment of in vitro encrustation stones. Scanning electron microscopy (SEM), energy dispersive X-ray spectroscopy (EDS), X-ray photoelectron spectroscopy (XPS) and $\mathrm{X}$-ray powder diffraction (XRD) analyses indicated a remarkable attenuation in encrustation occupation on the Re:IF-MoS 2 -coated catheter surfaces compared to neat catheters. The doped nanoparticles displayed a unique tendency to self-assemble into mosaic-like arrangements, modifying the surface to be encrustation-repellent. The mechanism of encrustation retardation on the surface coated catheters is discussed in some detail. The ramification of these results for the clogging of other body indwelling devices is briefly discussed.

Received 24th November 2013 Accepted 11th February 2014

DOI: $10.1039 / c 3 n r 06231 \mathrm{~g}$

www.rsc.org/nanoscale
A great variety of efforts to counteract the problem of encrustation have been reported, using many different strategies and approaches. ${ }^{\mathbf{1}}$ Nonetheless, so far the investigation has failed to eliminate it altogether.

The present work reports the mitigation of encrustation of a commercial all-silicone catheter by a surface coating of inorganic fullerene-like $\mathrm{MoS}_{2}\left(\mathrm{IF}-\mathrm{MoS}_{2}\right)$ nanoparticles.

Nanoparticles of inorganic layered compounds, such as $\mathrm{WS}_{2}$ and $\mathrm{MoS}_{2}$, are known to form a closed-cage fullerene-like structure. These nanoparticles were first reported in $1992^{\mathbf{1 0}, 11}$ and were discussed extensively in several review papers. ${ }^{12,13} \mathrm{An}$ SEM micrograph of a Re:IF-MoS 2 powder is shown in Fig. S1A. $\dagger$ The structure and shape of an individual IF- $\mathrm{MoS}_{2}$ nanoparticle is shown by the transmission electron microscopy (TEM) micrograph in Fig. S1B. $\dagger$ Each polyhedral multilayer nanoparticle is made of closed $\mathrm{MoS}_{2}$ layers, and its size is in the range of $50-200 \mathrm{~nm}$. Since an IF- $\mathrm{MoS}_{2}$ nanoparticle is a seamless closed-cage moiety, no structured-edges comprising plenty of dangling bonds are attendant. Hence, each nanoparticle is enveloped by van der Waals surfaces of fully saturated bonded (sulfur-terminated) atoms, possessing a low surface energy of $20 \mathrm{meV} \AA^{-2} \cdot{ }^{14}$ Therefore, the nanoparticles exhibit very low affinity to their environment and they can easily roll or slide. ${ }^{\mathbf{1 5}}$ Their unique atomically smooth topology (other than a few defects) and the fully saturated-bonds on their surfaces confer these nanoparticles (IF-WS ${ }_{2}$, IF-MoS ${ }_{2}$ ) superior solidlubrication behavior, ${ }^{\mathbf{1 6 - 1 8}}$ which has been recently exploited commercially.
Rehovot 76100 , Israel

${ }^{b}$ Department of Urology, Meir Medical Center, 59 Tchernichovsky street, Kfar Sava, 44410, Israel

'Sackler Faculty of Medicine, Tel-Aviv University, Tel-Aviv, Israel. E-mail: reshef. tenne@weizmann.ac.il; Tel: +972-8-9342394; +972-54-6244242

$\dagger$ Electronic supplementary information (ESI) available. See DOI: 10.1039/c3nr06231g 
Doping of the semiconducting IF- $\mathrm{MoS}_{2}$ nanoparticles with foreign atoms of rhenium $(<0.015$ at\%) endows them with $n-$ type conductivity by substitution of molybdenum atoms in the lattice. It is believed that the free electrons produced by the substantial rhenium doping are trapped by defects on the nanoparticle surface; so this extra charge is trapped at the surface or nearby. The Re-doped nanoparticles (Re:IF-MoS ${ }_{2}$ ) become negatively charged, thus repelling each other at close proximity. The rhenium doping was shown to have a profound effect on their physio-chemical behavior, rendering them a superior solid-lubricant. ${ }^{19}$ It was rationalized therefore that films of the electron rich Re:IF-MoS ${ }_{2}$ nanoparticles together with their superior solid-lubrication behavior ${ }^{\mathbf{1 6 - 1 8}}$ may attenuate encrustation development on catheter surfaces.

In the current study, a commercial all-silicone catheter was coated with a film of Re:IF-MoS $\mathrm{M}_{2}$ nanoparticles. A joint incubation of uncoated and Re:IF-MoS ${ }_{2}$-coated catheter specimens in an in vitro model of a catheterized urinary tract under encrustation conditions was followed. The nature and degree of the encrustation deposits which were developed on the uncoated and Re:IF-MoS ${ }_{2}$-coated catheter surfaces were comparatively assessed.

Herein, the interactions between encrustation concretions with Re:IF- $\mathrm{MoS}_{2}$ nanoparticles coating are studied in vitro. There exists no single standard for an in vitro model simulating encrustation. Thus, a variety of models for such experiments are reported in the literature. ${ }^{20}$ The use of artificially made urine provides a very convenient way for comparative studies of catheters coated with different surface functional groups. Moreover, human urine differs between one donor to another, and even between different micturitions of a single donor, thus favouring the use of a standard artificial urine source.

It is shown herein that the presence of a thin film of the Re:IF-MoS ${ }_{2}$ nanoparticles on the surfaces of the prosthetic device leads to a substantial attenuation in the encrustation on the catheter surface. It is believed that their atomically smooth, passivated-surface and negative surface charge of the nanoparticles delegate the device surface with low drag and adhesive characteristics, minimizing thereby the encrustation phenomena on the catheter surfaces. Hence, the Re:IF-MoS film coating would be expected to increase the indwelling durability and decrease associated morbidities to the patient.

\section{Results and discussion}

\section{Re:IF-MoS ${ }_{2}$ nanoparticles coating}

Fig. 1 shows photographs and SEM micrographs of uncoated (bare) and Re:IF-MoS ${ }_{2}$-coated catheter specimens. Native catheters had a transparent appearance (Fig. 1A) and their surfaces were found to be generally smooth with fine surface-dimples (inset of Fig. 1A). Fig. 1B demonstrates the successful application of the Re:IF-MoS ${ }_{2}$ nanoparticles on the catheter specimens by the production of a uniform gray coating layer. Although, detailed SEM inspections of the Re:IF-MoS ${ }_{2}$-coated catheters revealed that the coatings were not totally continuous on the entire catheter surface, i.e., small bald areas were observed within the coating films (inset of Fig. 1B). Furthermore, the

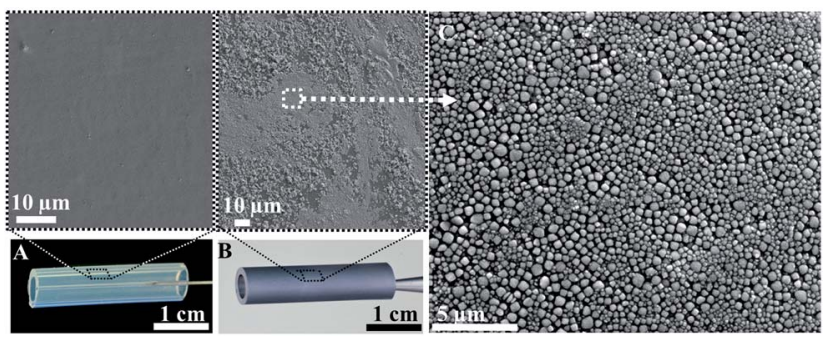

Fig. 1 Catheters before and after coating with the Re:IF-MoS 2 nanoparticles: (A) a photograph of a neat catheter specimen. The inset of (A) shows an SEM micrograph of the surface of an uncoated catheter. (B) A photograph of a Re:IF-MoS 2 -coated catheter specimen. The inset of (B) shows a low-magnification SEM micrograph of an Re:IF-MoS $2_{2}^{-}$ coated catheter. (C) A higher-magnification SEM micrograph of the Re:IF-MoS - -coated catheter surface (mode 1 ) which is marked in the inset of (B).

Re:IF-MoS $\mathrm{S}_{2}$ coating was characterized by bimodal domains. Most often, Re:IF-MoS ${ }_{2}$-coated domains displayed self-assembly of the nanoparticles into close-packed arrays with a mosaic-like appearance (mode 1) (Fig. 1C, and also Fig. 2 and S2A $\dagger$ ). It was noticed, however, that the mosaic-like pavement formed by the Re:IF-MoS $\mathrm{S}_{2}$ nanoparticles of catheter surfaces was very continuous and extended with increased periods of soaking in the well-deagglomerated (Re:IF-MoS $\mathrm{M}_{2}$ nanoparticles) coating's solution (Fig. 2). Additionally, certain Re:IF-MoS ${ }_{2}$-coated domains displayed a somewhat clumped arrangement (mode 2) of the nanoparticles (inset of Fig. 1B, and Fig. S2B and S3A $\dagger$ ). Remarkably, undoped IF-MoS $\mathrm{M}_{2}$ nanoparticles were appreciably more agglomerated and clumped (Fig. S3B $\dagger$ ), and consequently were not as effective in preventing encrustation as the doped nanoparticle coatings. Moreover, the Re:IF-MoS ${ }_{2}$-coating of the catheter surfaces exhibited a long-term robustness. It might be related to a mechanical interlocking of the nanoparticles on the catheter surfaces as a result of a fine swelling.

\section{Encrustation assessment}

Encrustation was gradually developed with time in the simulated urinary environment. After incubation periods of several hours, the initially clear urine solution was converted into a highly hazed appearance. This conversion indicated supersaturation of colloidal stones in the urinal medium, i.e. incidence of urea hydrolysis, ammonia release and its decomposition to $\mathrm{OH}^{-}$alkalizing ions. A compact accumulation of precipitates on the vessel's bottom and walls followed. The experiments were stopped at this point and the catheters were removed out of the solution. During the incubation period the $\mathrm{pH}$ of the urine solution increased from 6.4-6.5 to 7.5-7.6. Typically, the turbidity appears when the $\mathrm{pH}$ of the solution exceeds the value of $\sim 7$. Clearly, the lower the concentration of the enzyme, the longer it takes for the solution to become hazed. The urease enzyme which was utilized during the sets of experiments was found to potently catalyze the urea decomposition. A minor amount of enzyme (5 ppm) was added to the system in the beginning of each encrustation experiment in order to decelerate the process to a few hour periods. This time period is 


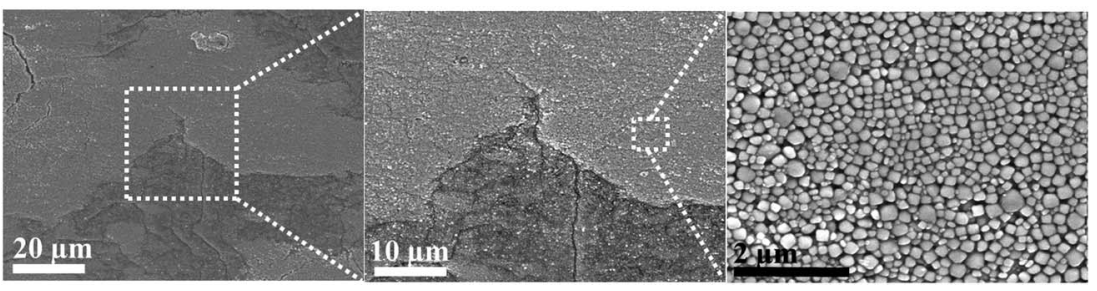

Fig. 2 SEM micrographs of a Re:IF-MoS ${ }_{2}$-coated catheter surface displaying a vast area on which the Re:IF-MoS 2 nanoparticles are selfassembled in a mosaic-like (mode 1 ) arrangement forming a close-packed film. The Re:IF-MoS 2 nanoparticles film is also continuous over this enlarged surface area.

nevertheless much shorter than that for in vivo encrustation. However, the current simulation employed severe conditions than the (slower) clinical situation.

Fig. 3 shows SEM micrographs of both uncoated (Fig. 3A and B) and Re:IF-MoS ${ }_{2}$-coated (Fig. 3C and D) catheter surfaces after an in vitro encrustation process (see also Fig. S2 †). The SEM micrographs are colored to facilitate a rapid discrimination between encrustation precipitates and the coating's nanoparticles. The insets of Fig. 3A and $\mathrm{C}$ display the original (without coloration) SEM micrographs for comparison. The SEM examination showed that, on both surface types, the solid precipitates were composed of two major morphologies: spherical precipitate with a perforated and poorly crystalline

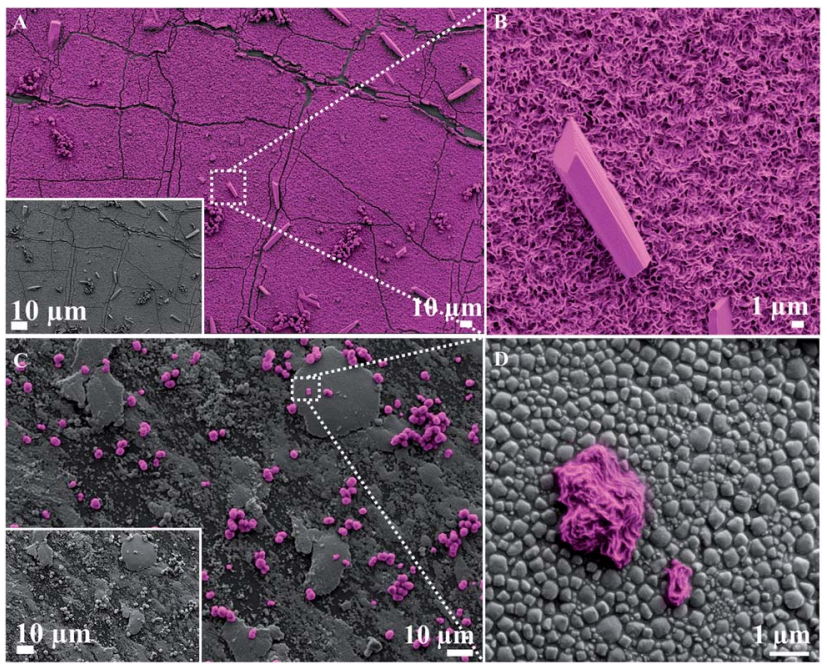

Fig. 3 SEM micrographs of encrusted, both uncoated and Re:IF-MoS 2 nanoparticle-coated, catheters after a joint in vitro encrustation process. The solid precipitants of encrustation are highlighted by a pink coloration to allow easy distinction between them to the Re:IF$\mathrm{MoS}_{2}$ nanoparticles. (A) The surface of a neat catheter specimen after its incubation in the encrustation model. Encrustation covers most of the surface forming a crust ("carpet"), on which elongated needle-like crystals are scattered. The original SEM micrograph is shown in the downright inset. The texture of the crust can be noticed by the highmagnification SEM micrograph in (B). (C) The surface of an encrusted catheter which was pre-coated by Re:IF-MoS 2 nanoparticles. The original SEM micrograph is shown in the downright inset. The sporadic growth of encrustation stones is displayed. A high-magnification SEM micrograph of the encrusted Re:IF-MoS 2 -coated catheter is shown in (D). structure and elongated needle-like crystals. In accordance with studies using similar artificial urine, ${ }^{21}$ the globular precipitates are reminiscent of hydroxyapatite ${ }^{21}$ and the elongated crystals are assigned as brushite.

Indeed, chemical analysis of the encrusted surfaces by EDS analyses confirmed the growth of calcium- and phosphoruscontaining stones. Fig. S4 $\uparrow$ exhibits the EDS spectra (accompanied by the respective SEM micrograph) which were generated from the two major typical morphologies of the grown deposits. Table 1 quantitatively summarizes the EDS results. A small magnesium quantity was observed in the spectra of the globularly shaped deposits. Nevertheless, struvite $\left(\mathrm{MgNH}_{4} \mathrm{PO}_{4} \cdot 6 \mathrm{H}_{2} \mathrm{O}\right)$ crystals were not observed in these series of experiments, most likely due to the low-basic urine $\mathrm{pH}$ in the current experimentation, which in most cases did not exceed 7.5. ${ }^{21}$ The experimentally observed $\mathrm{Mg}$ atoms are believed to be incorporated in the poorly crystalline apatite precipitate. The $\mathrm{Ca} / \mathrm{P}$ ratio in both the elongated crystals and the structureless stones was close to 1 . This ratio is typical of brushite whereas it deviates markedly from the composition of (fully crystallized) hydroxyapatite (1.6). As is further detailed below, comparatively XRD and XPS analyses of the surfaces of uncoated and Re:IF$\mathrm{MoS}_{2}$-coated catheter specimens also confirmed presentation of calcium-phosphate stones.

\section{The effect of Re:IF-MoS 2 nanoparticles on encrustation and its quantification}

Different growth and attachment modes of the encrusting solids were found on uncoated catheter samples (Fig. 3A and B) in comparison to those on Re:IF-MoS ${ }_{2}$-coated ones (Fig. 3C and D). These two specimens were incubated jointly in the same run, and this experiment was repeated great number of times revealing this difference reproducibly. Plain catheters were found to possess a continuous poorly crystalline crust ("carpet")

Table 1 EDS elemental composition of the two distinct morphologies of the in vitro encrustation deposits

Structural EDS results [at\%]

\begin{tabular}{lccccccc} 
Morphology & $\mathrm{C}(\mathrm{K})$ & $\mathrm{O}(\mathrm{K})$ & $\mathrm{Si}(\mathrm{K})$ & $\mathrm{P}(\mathrm{K})$ & $\mathrm{Ca}(\mathrm{K})$ & $\mathrm{Mg}(\mathrm{K})$ & $\mathrm{Ca} / \mathrm{P}$ \\
\hline Brushite & 11.8 & 65.2 & 0.7 & 11.9 & 10.4 & \multicolumn{1}{c}{} & 0.9 \\
Hydroxyapatite & 32.3 & 46.8 & 5.5 & 7.1 & 7.2 & 1.0 & 1.0
\end{tabular}


over the entire surface, consisting of fused globular stones (see also Fig. S2C and S4 $\dagger$ ). Both elongated and faceted crystals were observed scattered singly or as "flowers" with a common root in the center (Fig. S2C $\dagger$ ). In contrast (Fig. 3C and D), only a few and sporadically distributed stones occurred on the Re:IF- $\mathrm{MoS}_{2}$ coated catheters (see also Fig. S2A and $\mathrm{B} \dagger$ ). As was already mentioned, Fig. 3 displays the identical but colored SEM micrographs to enhance the discernment between encrustation stones and the Re:IF- $\mathrm{MoS}_{2}$ nanoparticles. A clear discrimination between the encrusted stones and the Re:IF-MoS ${ }_{2}$ nanoparticles usually requires application of higher SEM magnifications (Fig. 3D). Actually, the micrograph in Fig. 3C is not the most typical micrograph; the micrograph was chosen to be displayed since the encrustation growth phenomenon on Re:IF-MoS${ }_{2}$ coated catheters is hardly observable under low-magnification in the secondary electron (SE) mode. More commonly, however, encrustation deposits on most of the scanned areas in the analyzed Re:IF-MoS $\mathrm{M}_{2}$-coated catheters were much smaller and distant from one another. In fact, the nucleation density of encrustation was appreciably smaller on the Re:IF-MoS ${ }_{2}$-coated catheters compared to the encrusted uncoated catheter specimens. Importantly, although the Re:IF-MoS ${ }_{2}$ coating was not fully continuous or uniform, the degree of encrustation was greatly diminished on the entire catheter surface, i.e. even Re:IF-MoS ${ }_{2}$-uncoated areas on the catheter substrate were rarely encrusted. This fact may indicate that small patches of the encrustation film can be easily uprooted and washed away when the Re:IF-MoS ${ }_{2}$-coating is present on the catheter surfaces.

Time-dependent experiments showed that the amount of encrustation deposits increased with elongated incubation times for either the uncoated or Re:IF-MoS ${ }_{2}$-coated catheters. Longer incubation periods led to creation of thicker compact calcium phosphate films on the uncoated catheters. On the other hand, in the case of the Re:IF-MoS ${ }_{2}$-coated samples, new nuclei were not observed to be formed to a noticeable amount, but an enlargement of the already existing, sporadically distributed, calciumphosphate precipitates was observed with longer incubation time, mainly in the vertical direction (perpendicular to the substrate).

Using backscattering electron (BSE) imaging mode an enlarged surface area $\left(32000 \mu^{2}\right.$ ) over the encrusted Re:IF$\mathrm{MoS}_{2}$-coated catheter specimen could be carefully analyzed (see Materials and methods). The BSE mode was utilized since the deposited nanoparticles and encrusted stones were barely distinguishable using the SE detector at low magnifications (which could allow the examination of larger surface areas on each sample) (compare Fig. 4A and B). As seen in Fig. 4B, an evenly gray appearance represents the crust in the BSE-generated micrograph, which is much darker than the brighter graylevel of the nanoparticles. Fig. 4C-F exhibit verification of the fidelity of the much faster BSE analyses by a preceding EDSelemental mapping of the same area. Each (rather long scantime) EDS-map highlights the surface distribution of a single element of this sample. The congruence between the locations of stones as reflected from the Ca- and P-EDS maps and the BSE signal is clear. Thus, the BSE analysis revealed that the portion of the encrusted area over the enlarged surface $\left(32000 \mu \mathrm{m}^{2}\right)$ on the Re:IF-MoS ${ }_{2}$-coated catheter was $0.44 \%$ compared to $92.9 \%$ over a similar area of the uncoated catheter.

Table 2 presents an elemental-quantification by EDS of uncoated and Re:IF-MoS ${ }_{2}$-coated catheter surfaces after an encrustation process. The EDS analysis also found a considerable diminution of encrustation on the encrusted Re:IF- $\mathrm{MoS}_{2}$ coated specimen. Specifically, the Ca and P contents were 10.1 and 8.6 at\% (respectively) for the neat specimen against 1.2 and 1.9 at $\%$ for the Re:IF-MoS 2 -coated one. This result is consistent with the SE and BSE analyses.

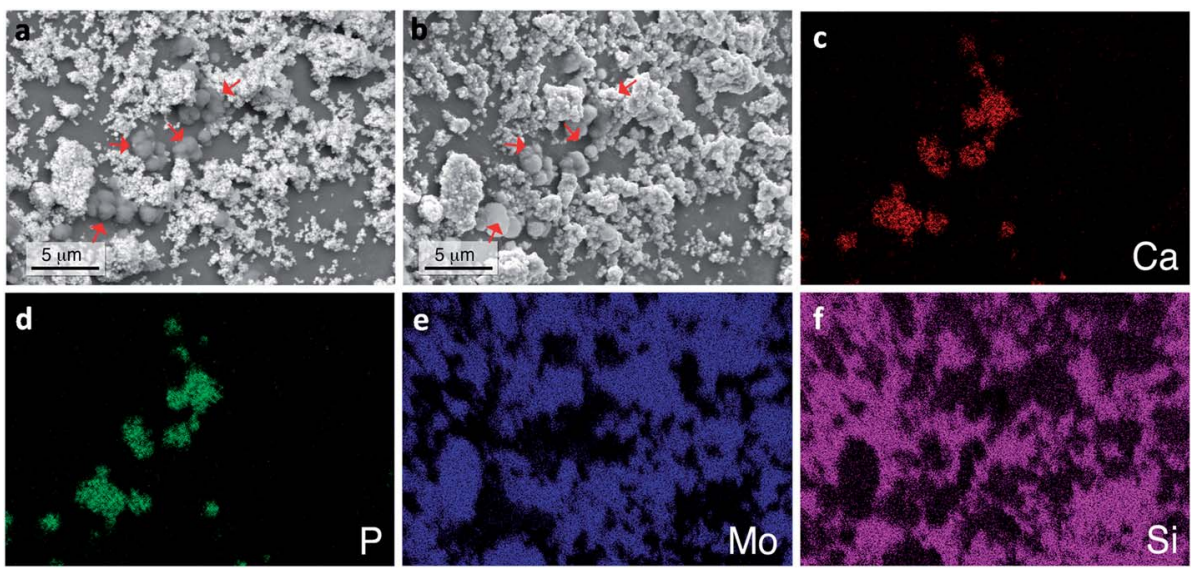

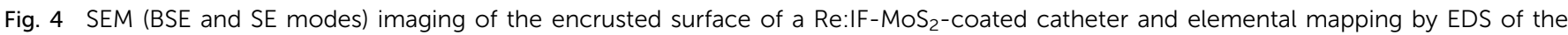
coincident surface. (A) SEM (BSE mode) and (B) SEM (SE mode) micrographs of the EDS-mapped area, with the stones delineated by red arrows. The BSE allows clear discrimination between the Re:IF-MoS ${ }_{2}$-nanoparticle coating and encrustation stones (see arrows) in (A). In contrast, as seen in (B), the contrast is insufficient to distinguish between the Re:IF-MoS 2 nanoparticles and the stones by the SE detector. This discrimination becomes prominent when small stones are to be identified. The EDS maps of (C) calcium and (D) phosphorus coincide with darker-gray imaged species (encrustation stones) in (A) while the map of (E), which highlights the molybdenum surface-distribution, is equivalent to the brighter-gray species (Re:IF-MoS 2 nanoparticles). (F) The silicone distribution map is also coincident with the darkest substrate-domains in (A). The conformity between the EDS maps and the SEM-BSE mode micrographs allows to rely on the fast BSE mode for the quantitative analysis over an enlarged $\left(32000 \mu \mathrm{m}^{2}\right)$ area, which cannot be performed by the slow and tedious EDS analysis. 
Table 2 Quantification by EDS of encrustation stones on pristine and encrusted (uncoated and Re:IF-MoS 2 -coated) catheters

EDS Encrustation quantification [at\%]

\begin{tabular}{lccccrrrr}
\hline Specimen & $\mathrm{C}(\mathrm{K})$ & $\mathrm{O}(\mathrm{K})$ & $\mathrm{Si}(\mathrm{K})$ & $\mathrm{Mo}(\mathrm{L})$ & $\mathrm{S}(\mathrm{K})$ & $\mathrm{P}(\mathrm{K})$ & $\mathrm{Ca}(\mathrm{K})$ & $\mathrm{Mg}(\mathrm{K})$ \\
\hline Pristine catheter & 35.9 & 31.7 & 32.4 & 0.0 & 0.0 & 0.0 & 0.0 \\
Encrusted uncoated catheter & 26.9 & 37.3 & 16.5 & 0.0 & 0.0 & 8.6 & 10.1 \\
Encrusted IF-coated catheter & 28.9 & 20.5 & 19.2 & 10.7 & 17.0 & 1.9 & 1.2 & 0.6 \\
\hline
\end{tabular}

A couple of catheter specimens were additionally studied by XPS. Fig. 5A presents the broad-scans of encrusted, Re:IF- $\mathrm{MoS}_{2}$ coated and uncoated, catheters, together with the highly

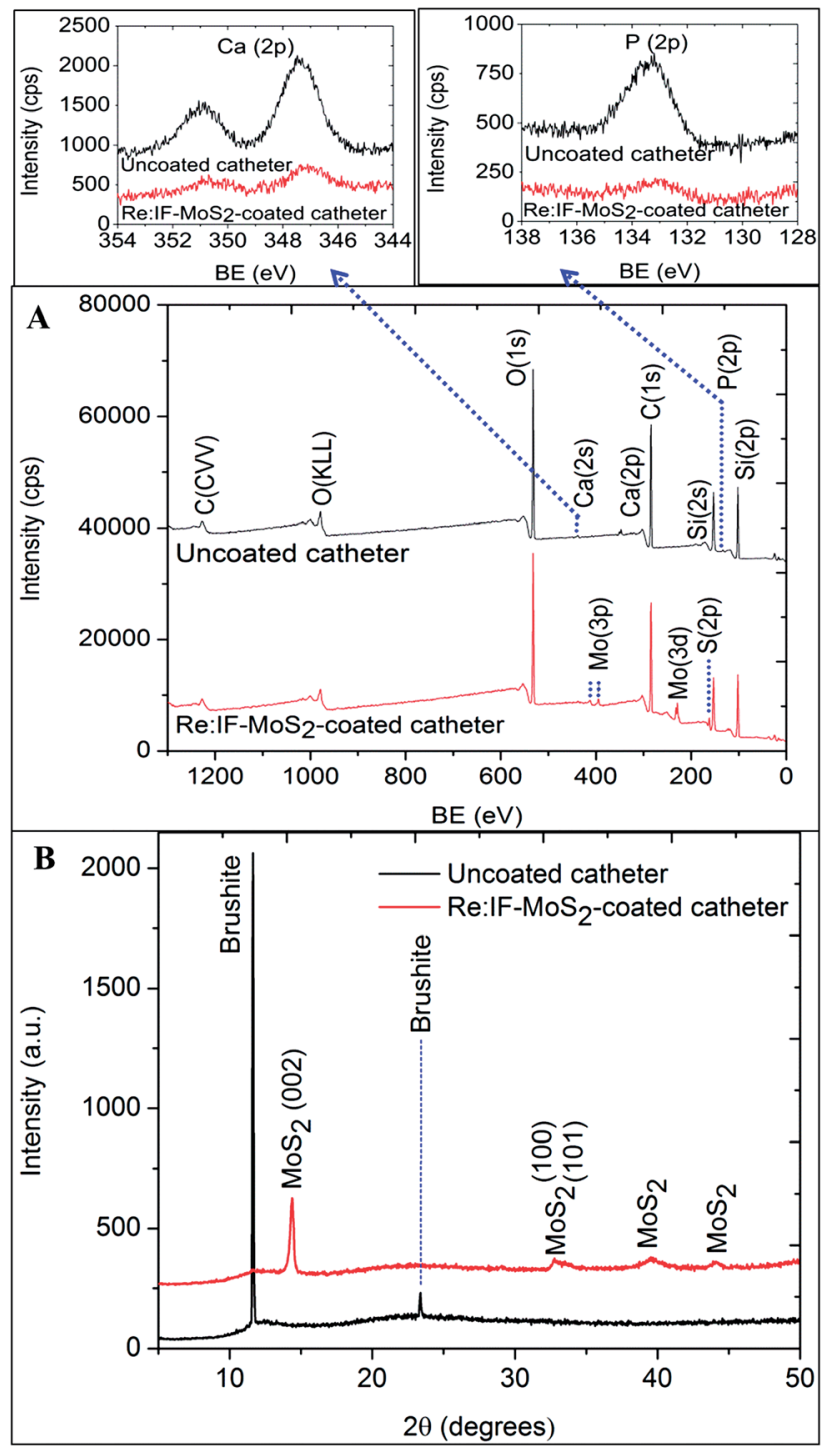

Fig. 5 XPS and XRD spectra of uncoated and Re:IF-MoS $\mathrm{S}_{2}$-coated catheter specimens after a joint incubation in the encrustation model. (A) XPS broad-scans and highly resolved binding energy peaks of calcium (2p) and phosphorus (2p) in the upper insets. (B) XRD spectra of the encrusted catheter specimens confirmed the presence of calcium-phosphate deposits on the uncoated surfaces while for the Re:IF-MoS 2 -coated surface, the encrustation signals were smaller than the detection limit $(0.5 \mathrm{wt} \%)$ of the instrument. resolved binding energy peaks of $\mathrm{Ca}(2 \mathrm{p})$ and $\mathrm{P}(2 \mathrm{p})$. Detailed atomic percent composition of the catheter surfaces achieved by the XPS measurements is presented in Table 3. The pronounced difference in the contents of encrustation-related elements is clearly displayed by the XPS results: the atomic concentrations of $\mathrm{Ca}(2 \mathrm{p})$ and $\mathrm{P}(2 \mathrm{p})$ for the uncoated catheter were 0.74 and 0.99 at\%, respectively. The concentration of these atoms was reduced to 0.21 and 0.15 at $\%$ for the Re:IF- $\mathrm{MoS}_{2}$-coated catheter. As expected, the atomic percent ratio of Mo to $\mathrm{S}$ on the Re:IF-MoS ${ }_{2}$-coated specimen was 0.5 .

XRD measurements (Fig. 5B) of the encrusted uncoated specimen identified two intense peaks with $2 \theta$ values of 11.6 and 23.4 degrees, which can be assigned to the calcium-phosphate phase of brushite (black spectrum in Fig. 5B). In accordance with previous works, ${ }^{21,22}$ the poorly crystalline hydroxyapatite could not be identified in this pattern. The XRD pattern of the encrusted Re:IF-MoS ${ }_{2}$-coated catheter exhibited the $\mathrm{MoS}_{2}$ peaks, while the brushite phase had largely vanished, confirming thereby the level of encrustation on this substrate which was under the detectable amount (0.5 wt\%).

The experimental results principally demonstrate that the self-assembled Re:IF-MoS ${ }_{2}$ nanoparticle film has a clear attenuating effect on the encrustation of all-silicon catheters.

Stones of similar types were detected on both the uncoated and Re:IF-MoS $\mathrm{M}_{2}$-coated catheter samples. Therefore, the pres-

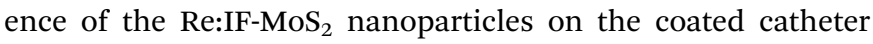
specimens influenced neither the morphology nor the chemical composition of the in vitro encrustation. However, the difference in the degree of encrustation was consistently detected regardless of the technique used for the analysis.

The exact mechanism of the encrustation suppression on Re:IF-MoS ${ }_{2}$-coated catheters is not fully comprehensible, yet. Nevertheless, a few key physio-chemical properties of these nanoparticles might provide guidelines for this mechanism. Particularly, their charge, low surface free energy and nanotexture are unique properties which are delegated to the coated catheter surface. Therefore, the presence of the nanoparticle film on the catheter surface alters its nanostructure, as well as its chemistry, influencing thereby the physio-chemical characteristics of the catheter surface.

Two different encrustation mechanisms can be considered; one of them involves a direct nucleation of the hydroxyapatite and brushite at stable surface-sites enabling their further growth on the available area. Simultaneously, stones nucleate and grow in the solution and subsequently these colloidal nanoparticles precipitate/adhere on the catheter surface. 
Table 3 XPS elemental composition of encrusted and non-encrusted, both uncoated and Re:IF-MoS ${ }_{2}$-coated, catheter specimens

\begin{tabular}{|c|c|c|c|c|c|c|c|c|c|}
\hline \multicolumn{10}{|l|}{ XPS results [at\%] } \\
\hline & Re:IF-MoS ${ }_{2}$-coated & - & - & 20.30 & 20.47 & 43.28 & 6.21 & 6.49 & 3.13 \\
\hline Encrusted specimens & Uncoated & 0.74 & 0.99 & 22.75 & 24.26 & 51.25 & - & - & - \\
\hline & Re:IF-MoS ${ }_{2}$-coated & 0.21 & 0.15 & 22.17 & 23.07 & 48.83 & 2.13 & 2.31 & 1.15 \\
\hline
\end{tabular}

The observed massive supersaturation in the urine during encrustation experiments indicates the enormous amount of colloidal stones surrounding each incubated catheter specimen. This situation introduces an abundant possibility of stone precipitation and adherence. However, as investigation of the surface-structure of the Re:IF-MoS 2 -coated catheters showed, the colloidal stone particles approaching a catheter specimen from the bulk solution encounter an architecture totally different from the smooth substrate of a neat catheter specimen. Generation of the special surface-nanostructure by self-assembly of the negatively charged Re:IF-MoS ${ }_{2}$ nanoparticles into two-dimensional close-packed arrays (Fig. 1C, 2 and $\mathrm{S} 3 \mathrm{~A} \dagger$ ) produced a compact dense array of nano-distant bumps. This nanoparticle arrangement might reduce the availability of contact-points for anchoring stones onto the Re:IF-MoS ${ }_{2}$-coated catheter surface, which is an established mechanism for self-cleaning surfaces. ${ }^{23-25}$

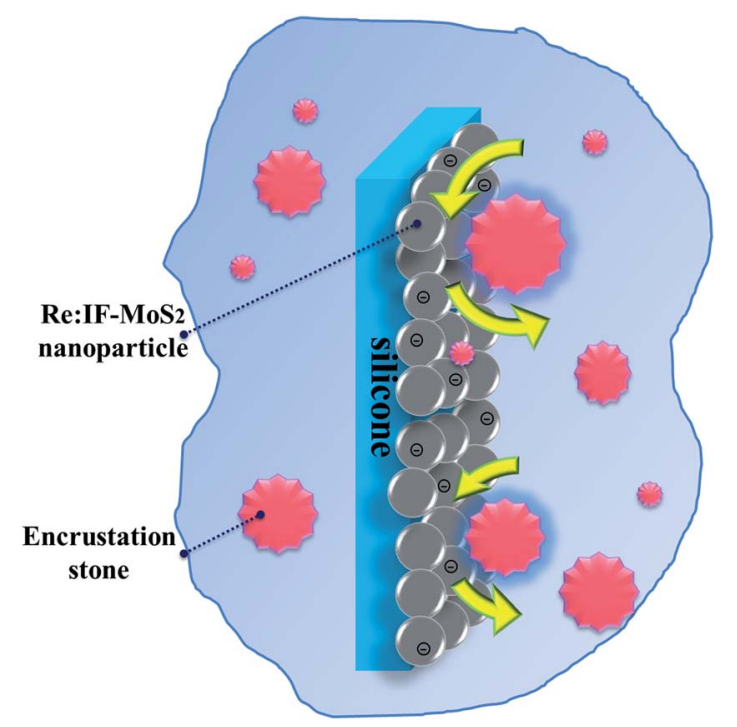

Fig. 6 A schematic illustration of a catheter surface which is coated by the Re:IF-MoS 2 nanoparticles in a urine environment under encrustation conditions. By self-assembly, the negatively charged nanoparticles produce a surface architecture which is close-packed and nano-bumped with an arrangement resembling a mosaic pavement. This highly-ordered and closely-packed film, together with the lowaffinity of the nanoparticles towards their environment (closed-caged moieties with minor dandling bonds), their low surface energy and their superior lubrication behavior (low friction), are all conductive to weak anchoring of the stones to the catheter surface. Thus, encrustation stones are believed to slip from the surface while approaching it.
Additionally, due to its atomically smooth surface and low surface energy, ${ }^{14}$ the Re:IF-MoS ${ }_{2}$ material is known to be chemically very inert ${ }^{26,27}$ and induce very low friction. ${ }^{16-18}$ Therefore, the anchoring potential of the hydroxyapatite colloidal nanoparticles to the underlying substrate is expected to be very low. Consequently, the stones are believed to "slip" on the Re:IF$\mathrm{MoS}_{2}$-coated catheters once approaching the surface (Fig. 6).

The visual absence of encrustation on bald areas on the Re:IF-MoS ${ }_{2}$-coated catheter surfaces suggests that, even if some encrustation has occurred on these areas, these patches could be easily uprooted by the dynamic flow of the urine.

The specific structure and chemistry of each Re:IF-MoS nanoparticle provides further support to the above model; the low surface energy $\left(20 \mathrm{meV} \AA^{-2}\right)^{14}$ of the basal (0001) $2 \mathrm{H}-\mathrm{MoS}_{2}$ surface implies that the terminal (sulphur) atoms are very inert with respect to a specific chemical reaction under the present conditions. ${ }^{26,27}$ However, the curved (0001) surfaces of the IF-MoS nanoparticles contain a small amount $(<5 \%)$ of structural defects, which are chemically reactive, but can be passivated via adsorption of specific moieties. ${ }^{28}$ Such defects can be the source of the rather rare and random growth of stones on the surface coated catheters.

Future experiments are aimed at investigating the influence of such Re:IF-MoS ${ }_{2}$ nanoparticles coatings on indwelling medical devices of different sorts, like blood stents, or metallic implants. The biocompatibilities of the IF-MoS 2 nanoparticles are currently being investigated. Early indications suggest that these nanoparticles are benign and do not pose any toxic risk. ${ }^{29}$

\section{Conclusions}

A new approach is presented to reduce encrustation of silicone catheters by the application of Re:IF-MoS ${ }_{2}$ nanoparticles. It is demonstrated that the negatively charged surface Re:IF-MoS nanoparticles produce coating films comprised of long-range domains in which the Re:IF-MoS 2 nanoparticles are selfassembled into a mosaic-like order, showing a relatively small tendency to agglomerate. The results of this work suggest that the presence of thin coatings of Re:IF- $\mathrm{MoS}_{2}$ nanoparticles on the surface of silicone catheters effectively attenuates the growth and attachment of encrustation concretions.

\section{Materials and methods}

\section{Coating catheter specimens by Re:IF-MoS ${ }_{2}$ nanoparticles}

Specimens of a commercial all-silicone medical-grade 2-way Foley catheter (Hangzhou Fushan Medical Appliances Co. Ltd., 
China; supplied by: J.S Gull Ltd., Israel), 22 French size $(7.3 \mathrm{~mm})$ and $400 \mathrm{~mm}$ long, were employed throughout all the described experiments. Segments of $3 \mathrm{~cm}$ length were cut from the cylindrical shaft of the device and then were cut along the longitudinal axis. The external (convex) surfaces of the catheter specimens were used for carrying out all the reported analyses.

A suspension of $0.05 \mathrm{wt} \%$ Re:IF-MoS ${ }_{2}$ nanoparticles in ultrapure $\mathrm{H}_{2} \mathrm{O}$ (Milli-Q RG, Millipore) was sonicated, using an ultra-sonic probe mixer (Vibra Cell VCX400, $400 \mathrm{~W}$, Sonics \& Materials) for $30 \mathrm{~min}$. The ultra-sonication was alternately applied (6 $\mathrm{s}$ activation and $4 \mathrm{~s}$ deactivation) on the Re:IF-MoS ${ }_{2}^{-}$ suspension, during which a constant magnetic stirring was implemented.

Catheter specimens were individually suspended in vials containing $10 \mathrm{ml}$ of the Re:IF-MoS $\mathrm{M}_{2}$ suspension. The vials were left 24 hours for mixing using a rotation machine. Prior to the analyses, the catheter specimens were removed from the Re:IFMoS2-suspension and were rinsed with ultrapure $\mathrm{H}_{2} \mathrm{O}$. Moreover, for the sake of comparison, uncoated bare catheter specimens were put inside similar vials which contained $10 \mathrm{ml}$ $\mathrm{H}_{2} \mathrm{O}$, and were treated through the same procedure. Additional series of samples were prepared from pristine silicone catheters for reference purposes. These samples were analyzed without any prior treatment.

\section{In vitro encrustation process}

An in vitro encrustation process was conducted using a custombuilt model of a catheterized-like urinary tract. The simulated stress results in precipitation of in vivo-like encrustation deposits under defined, controlled and reproducible conditions. However, the incubation time-period in this study is much shorter and the rate of incidence of encrustation is much accelerated in comparison to those in the human-body. ${ }^{1,2}$ As was already pointed out, there exists no single standard for an in vitro test model for such experiments. Thus, a variety of models were reported introducing different fundamental experimental parameters. ${ }^{20}$ The present model has been used extensively in the past.

In the present work, the encrustation processes were performed in a glass reaction vessel equipped with a fitting lid within which 12 marked stainless steel rods were equally positioned. At the end of each rod stood a hook on which a single vertical specimen was positioned. The vessel was placed in an incubator to maintain the physiological temperature $\left(37^{\circ} \mathrm{C}\right)$. In order to systematically reproduce the conditions of the encrustation process along the experiments, an artificial urine solution was used. This solution has a well-defined composition, compared to the human urine which has a non-uniform composition among different native donors and within different micturitions of an individual donor. The artificial urine solution used in the present work was similar to that used by Griffith $e t$ al. ${ }^{30}$ The solution consisted of 10 solutes (see Table $\mathrm{S} 1, \dagger$ initial $\mathrm{pH}=6.4-6.5$ ), whose concentrations were equivalent to the average concentration found over a 24 hour period in the urine of normal human. ${ }^{30}$ Alkalinization of the urinal medium was triggered here by a direct addition of a Jackbeanderived urease (type III, Sigma-Aldrich). The urease was supplied as a lyophilized powder, which was dissolved in a preprepared filtered $(0.45 \mu \mathrm{m})$ sodium phosphate buffer $(2 \mathrm{M}, \mathrm{pH}=$ 7.0). In most of the reported experiments, the urease concentrations and incubation times were approx. $0.05 \mathrm{mg}$ per $100 \mathrm{ml}$ (5 ppm) urine solution and approximately 8-12 hours, respectively. The enzyme powder was found to be very hygroscopic, affecting the actual enzyme solution concentrations. Therefore, it is important to emphasize that the incubation period, i.e. the time lapse for turbidity, varied somewhat from one series of measurement to the other, depending on the freshness of the enzyme powder which is very hygroscopic. Thus, care was taken to maintain the enzyme under strictly dry conditions. Furthermore, during all sets of experiments, the two kinds of specimens (Re:IF-MoS ${ }_{2}$-coated and uncoated) were simultaneously incubated in the encrustation reactor. The samples were taken out for analysis as soon as the solution lost its full transparency and became massively turbid. Upon removal of the encrusted specimens out of the in vitro model, they were gently rinsed with ultrapure $\mathrm{H}_{2} \mathrm{O}$ to remove loosely attached debris and were stored inside an evacuated desiccator till further analyses. Furthermore, during each experiment $\mathrm{pH}$ measurements were carried out by a pH-meter (pH510, Eutech Instruments).

\section{SEM and EDS analyses}

SEM (model Ultra 55 FEG Zeiss; LEO model Supra 55 vp, Carl Zeiss International, Oberkochen, Germany, and E-SEM-FEG XL30 Philips/FEI) was used for this study. The SEM set-ups were operated in either SE or BSE modes.

EDS (EDAX instrument, Phoenix, attached to the E-SEM) was used for the chemical analysis of the specimens. Here, two modes of work were implemented for the chemical analysis. In the first one, the beam was focused on a single stone (high magnification). The analysis was repeated three times for stones of the same morphology and the result is reported as an average of the three measurements. In addition, a global (low magnification) EDS analysis of the encrusted surface $\left(3000 \mu \mathrm{m}^{2}\right)$ was carried out. The EDS analyses of bare, encrusted, uncoated and Re:IF-MoS $\mathrm{S}_{2}$-coated catheter specimens were compared. All the low magnification EDS analyses were performed by sampling three distinct surface locations. The results are reported as the average of the three EDS measurements. The accelerating voltage of the beam for the EDS analysis was limited to $15 \mathrm{keV}$.

SEM imaging and image analysis were used to perform quantitative analysis of the encrustation developed on the catheter surface. Imaging the catheter surface with the SE detector proved to be rather problematic for this purpose. Discrimination between the precipitated stones and the Re:IF-MoS ${ }_{2}$ nanoparticles was effective at high magnification $(\times 20000)$, only. However, the heterogeneity of the surface did not permit acquiring sufficient data for a fully quantitative analysis under high magnification. Conversely, at low magnification the discrimination between the stones and the Re:IF- $\mathrm{MoS}_{2}$ nanoparticles was not adequate in the SE mode. Therefore, mapping the encrusted surface with a BSE detector, which is sensitive to the atomic number $(Z)$, combined with image analysis was preferred for the quantitative analysis. The contrast difference in 
the BSE mode allowed clear discrimination among the encrusted

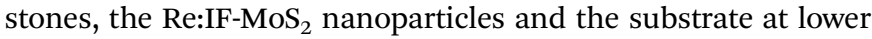
magnification $(\times 5000)$ and over large surface areas, thus enabling quantitative analysis of the different substrates.

Confirmation of the BSE mapping with EDS analysis, which is slow and rather tedious, was done with full agreement between the two analyses. The surface area of a Re:IF-MoS nanoparticle-coated specimen after encrustation was analyzed by dividing the surface into a raster (mesh). Each raster unit was scanned by the BSE detector at a magnification of $\times 5000$ (50 micrographs, $640 \mu \mathrm{m}^{2}$ each, $32000 \mu \mathrm{m}^{2}$ total area). Image analysis of the BSE data was done using the Image (National Institutes of Health, USA) software.

Samples for the SEM analyses were prepared in the following manner: $\sim 5 \times 5 \mathrm{~mm}^{2}$ samples were cut from the middle of each parent sample. A thin layer of gold-palladium was evaporated on each specimen using a high vacuum evaporation set-up (S150 sputter coater, Edwards). For the EDS analysis, carbon evaporation was applied instead, using a high vacuum evaporation set-up (BOC FL400, Edwards).

\section{XPS measurements}

The XPS measurements were carried-out with a Kratos AXIS ULTRA system, operating in a ultra-high $\left(10^{-9}\right.$ torr $)$ vacuum. A monochromatized $\mathrm{Al}(\mathrm{K} \alpha)$ X-ray source $(h \nu=1486.6 \mathrm{eV})$ at $75 \mathrm{~W}$ and detection pass energies ranging between 40 and $80 \mathrm{eV}$ were used. The data were recorded at a take-off angle of $0^{\circ}$ with respect to the surface normal. A low-energy electron flood gun (eFG) was applied for charge neutralization. The binding energy scale was referenced to the main $\mathrm{C}(1 \mathrm{~s})$ peak attributable to a hydrocarbon at $284.9 \mathrm{eV}$.

To minimize the beam damage effects, the analysis time was set as $30 \mathrm{~min}$. Curve fitting analysis was based on linear background subtraction and application of Gaussian-Lorenzian line shapes. Quantification was carried-out using the peak area, and corrected with Scofield sensitivity factors. Signals were collected from an area size of $900 \times 400 \mu \mathrm{m}^{2}$ for each sample.

In addition to the encrusted specimens, a few non-encrusted samples (prior to urine exposure) for control were also analyzed including: Re:IF-MoS ${ }_{2}$-coated and also bare untreated catheter specimens.

\section{XRD measurements}

The XRD measurements were carried out in reflection geometry using a diffractometer (TTRAX III Rigaku, Japan) equipped with a rotating $\mathrm{Cu}$ anode operating at $50 \mathrm{kV}$ and $200 \mathrm{~mA}$ and with a scintillation detector. $\theta / 2 \theta$ scans were performed under specular conditions in Bragg-Brentano mode with variable slits. The samples were scanned from 5 to $50^{\circ}$ of $2 \theta$ with a step size of $0.025^{\circ}$ and a scan speed of $0.4^{\circ}$ per minute. Phase analysis was performed using the Jade 9.1 software (Materials Data, Inc.) and PDF-4+ 2010 database (ICDD).

\section{Acknowledgements}

We are grateful to Dr Y. Feldman (XRD); Dr H. Cohen and T. Bendikov (XPS); L. Yadgarov (chemical analysis of the Re:IF-
$\mathrm{MoS}_{2}$ nanoparticles); Dr E. Kartvelishvily and E. Klein (SEM, EDS); Dr A. Baram (image analysis). RT is supported by the Israel Science Foundation; ERC INTIF 226639 grant; ERC-PoC 324571 grant; Harold Perlman and the Irving and Azelle Waltcher Foundations, and the Irving and Cherna Moskowitz Center for Nano and Bio-Nano Imaging. He holds the Drake Family Chair in Nanotechnology and is the director of the Helen and Martin Kimmel Center for Nanoscale Science.

\section{References}

1 T. M. Hamill, B. F. Gilmore, D. S. Jones and S. P. Gorman, Expert Rev. Med. Devices, 2007, 4, 215-225.

2 D. J. Stickler, Nature, 2008, 5, 598-608.

3 D. J. Stickler and R. C. L. Feneley, Spinal Cord, 2010, 48, 784790.

4 G. L. Shaw, S. K. Choong and C. Fry, Urol. Res., 2005, 33, 1722.

5 M. Sofer and J. D. Denstedt, Curr. Opin. Urol., 2000, 10, 563569.

6 A. W. S. Elves and R. C. L. Feneley, Br. J. Urol., 1997, 80, 1-5. 7 J. D. Denstedt, T. A. Wollin and G. Reid, J. Endourol., 1998, 12, 493-500.

8 S. K. Choong and H. N. Whitfield, J. Endourol., 2000, 14, 1923.

9 D. Stickler, R. Young, G. Jones, N. Sabbuba and N. Morris, Urol. Res., 2003, 31, 306-311.

10 R. Tenne, L. Margulis, M. Genut and G. Hodes, Nature, 1992, 360, 444-446.

11 L. Margulis, G. Salitra, R. Tenne and M. Talianker, Nature, 1993, 365, 113-114.

12 R. Tenne, Nat. Nanotechnol., 2006, 1, 103-111.

13 C. N. R. Rao and A. Govindaraj, Adv. Mater., 2009, 21, 42084233.

14 T. Björkman, A. Gulans, A. V. Krasheninnikov and R. M. Nieminen, Phys. Rev. Lett., 2012, 108, 235502-235506.

15 O. Tevet, P. Von-Huth, R. Popovitz-Biro, R. Rosentsveig, H. D. Wagner and R. Tenne, Proc. Natl. Acad. Sci. U. S. A., 2011, 108, 19901-19906.

16 L. Rapoport, Y. Bilik, Y. Feldman, M. Homyonfer, S. R. Cohen and R. Tenne, Nature, 1997, 387, 791-793.

17 M. Chhowalla and G. A. Amaratunga, Nature, 2000, 407, 164167.

18 O. Goldbart, O. Elianov, D. Shumalinsky, L. Lobik, S. Cytron, R. Rosentsveig, H. D. Wagner and R. Tenne, Nanoscale, 2013, 5, 8526-8532.

19 L. Yadgarov, R. Rosentsveig, G. Leitus, A. Albu-Yaron, A. Moshkovich, V. Perfilyev, R. Vasic, A. I. Frenkel, A. N. Enyashin, G. Seifert, L. Rapoport and R. Tenne, Angew. Chem., Int. Ed., 2012, 51, 1148-1151.

20 M. M. Tunney, D. S. Jones and S. P. Gorman, Methods Enzymol., 1999, 310, 558-566.

21 F. Grases, A. I. Villacampa, O. Söhnel, E. Königsberger and P. M. May, Cryst. Res. Technol., 1997, 32, 707-715.

22 A. J. Cox, J. E. Harries, D. W. L. Hukins, A. P. Kennedy and T. M. Sutton, Br. J. Urol., 2008, 59, 159-163.

23 A. Marmur, Langmuir, 2004, 20, 3517-3519. 
24 T. S. Wong, S. H. Kang, S. K. Y. Tang, E. J. Smythe, B. T. Hatton, A. Grinthal and J. Aizenberg, Nature, 2011, 477, 443-447.

25 L. Feng, Y. Zhang, J. Xi, Y. Zhu, N. Wang, F. Xia and L. Jiang, Langmuir, 2008, 24, 4114-4119.

26 O. Lang, R. Schlaf, Y. Tomm, C. Pettenkofer and W. Jaegermann, J. Appl. Phys., 1994, 75, 7805-7813.

27 W. Jaegermann and H. Tributsch, Prog. Surf. Sci., 1988, 29, 1167.
28 C. Shahar, D. Zbaida, L. Rapoport, H. Cohen, T. Bendikov, J. Tannous, F. Dassenoy and R. Tenne, Langmuir, 2009, 26, 4409-4414.

29 (a) H. Wu, R. Yang, B. Song, Q. Han, J. Li, Y. Zhang, Y. Fang, R. Tenne and C. Wang, ACS Nano, 2011, 5, 1276-1281; (b) M. Pardo, T. Shuster-Meiseles, S. Levin-Zaidman, A. Rudich and Y. Rudich, Environ. Sci. Technol., 2014, 48, 3457-3466.

30 D. P. Griffith, D. M. Musher and C. Itin, Invest. Urol., 1976, 13, 346-350. 